\title{
High-Altitude UAS Pseudo-Satellites: Architecture for End-to-End Military Communications
}

\author{
Harry Lyon ${ }^{1}$ and Gokhan Inalhan ${ }^{2}$ \\ Cranfield University, Cranfield, Bedfordshire, MK43 0AL, United Kingdom \\ Daniel Bourne ${ }^{3}$ \\ Airbus Defence and Space Ltd, Portsmouth, PO3 5PU, United Kingdom \\ Antonios Tsourdos ${ }^{4}$ \\ Cranfield University, Cranfield, Bedfordshire, MK43 0AL, United Kingdom
}

\begin{abstract}
High-Altitude Pseudo-Satellites (HAPS) have been identified as a potential option to either supplement or replace various military communications services. A network of HAPS aircraft operating at an altitude of $20 \mathrm{~km}$ offers localized, high performance services to military operations. The intention of this work is to investigate whether a network of HAPS (specifically Airbus' Zephyr $S$ platform) is preferable to that of standard military communications infrastructure. Individual technologies were not studied directly, but rather the overall services were analyzed. This study will not replace services one-for-one, but rather investigate how HAPS can augment capabilities of current infrastructure. This need for supplementation of services may arise from increased service demand, or in the case of emergency, where other systems may be compromised. A particular emphasis is placed on command and control (C2) of the aircraft, and how this can be harnessed to produce the required communications network.
\end{abstract}

\section{Nomenclature}

$A N L \quad=$ Aerial Network Layer

$C 2=$ Command and Control

$C I S=$ Communication and Information Services

GT $\quad=$ Ground Terminal

HAPS $=$ High-Altitude Pseudo-Satellite

IPL $=$ Inter-Platform Link

$M o D=$ Ministry of Defence

NEC $\quad$ Network Enabled Capability

OSC $=$ Offensive Space Control

$Q o S=$ Quality of Service

RSS $\quad=$ Received Signal Strength

\footnotetext{
${ }^{1}$ M.Sc. Student, School of Aerospace, Transport and Manufacturing

${ }^{2}$ BAE Systems Chair, Professor of Autonomous Systems and Artificial Intelligence, Cranfield University and AIAA Associate Fellow.

${ }^{3}$ Graduate RF \& Microwave Hardware Detailed Design Engineer, RF Production Definition

${ }^{4}$ Head of Autonomous and Cyber-Physical Systems Centre, Centre for Autonomous and Cyber-Physical Systems and AIAA Senior Member.
} 


\section{Introduction}

The correct use of information systems has been identified as critical to operational success. The combination of effective sensors, reliable and fast networks, as well as competent decision making has been highlighted as the cornerstones of gaining an informational advantage in the battlespace [1]. Current convention dictates that a Communication and Information System (CIS) is comprised of space and terrestrial architectures that enable the distribution of information across varied operational areas.

It has been long understood that a space segment has great utility, enabling a persistent global presence that isn't bound by global litigation of normal airspace [2]. However, a wide variety of challenges arise from the utilization of these systems; the space segment utilizes satellites occupying geosynchronous orbits[3]. By following a predictable orbit, satellite systems are susceptible to hostile Offensive Space Control (OSC) operations that can temporarily or even permanently damage the system via cyber warfare or direct ascent systems [4]. Although effective active and passive countermeasures (such as radioactive shielding and 'Retaliatory Active Measures) [5] have been employed, the nonzero risk of disruptions to vital satellite communication services suggest a need for other external mitigation strategies. There are numerous other disadvantages, such as development and operational cost, alongside security risks associated with sharing bandwidth with other commercial services. From a network perspective, the airspace medium has been somewhat unnecessarily overlooked.

'Air Power' provides significant benefits over nominal ground and satellite-based services. The 'Height, Speed and Reach' [6] aspects of Air Power offer great advantages over terrestrial systems. The region-specific applications of Air Power offer a mitigation strategy to the various security risks associated with shared military-commercial SATCOM services. Current Air Power capabilities do not currently include communications networks as associated advantages, and admittedly historically it doesn't benefit from the 'persistence' [6] of terrestrial and satellite services. HAPS platforms have been identified as an enabling technology that benefits from both the flexibility of Air Power, with the potential persistence of currently enjoyed by 'Space' and 'Land Power'.

In this work, we investigate the validity of using HAPS technology for military communications by using the Airbus Zephyr Platform. Our analysis is based on UK military communications infrastructure as a baseline for comparison and the operation doctrine of the UK military and NATO. For example, in the advent of an emergency (due to increased data traffic, or disruption of satellite communications), HAPS has been identified as a technology that could replace the beyond line of sight communication services that Skynet nominally provides. By deploying a network of HAPS aircraft, regional communications can be upheld for a period of time while SATCOM services are restored.

In this work, rather than making like-for-like a comparison of the individual technology available, a 'HAPS Communications Service Package' is produced, which supplements some of the capabilities enabled by current infrastructure (Figure 1). This service package is then applied to a specific operational case study, whereupon performance can then be compared to existing CIS infrastructure. This is achieved via a series of predefined qualitative and quantitative parameters as given in Section IV. 


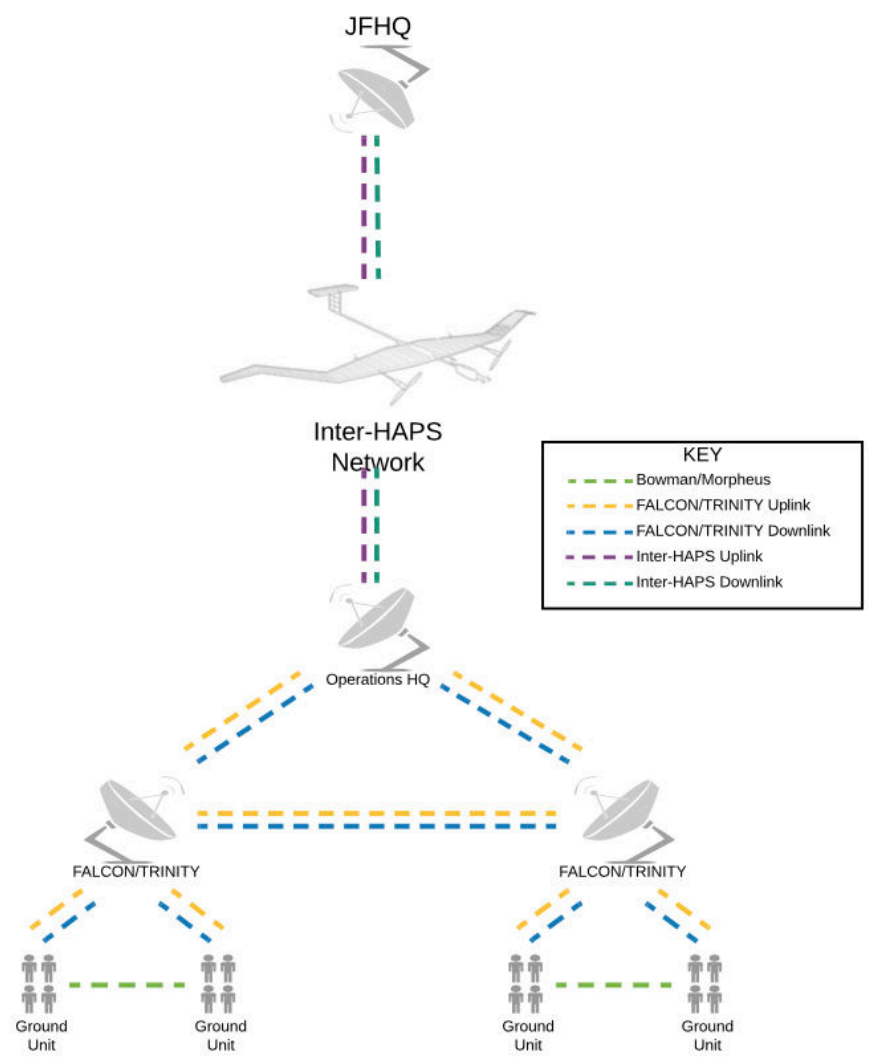

Figure 1: Schematic of how HAPS will fit into the current network architecture

The remainder of the paper is structured as follows:

Section II will provide an overview of the current systems employed by the UK military, giving an insight to the infrastructure currently employed, as well as detailing the chosen HAPS platform for this study. Section III will detail the service package itself, starting the network architecture and payload, then detailing the $\mathrm{C} 2$ and handover strategy. highlighting the architecture and C2 options that will be employed. Finally, Section IV applies the HAPS communications network to a fictional case study, where aspects of the network is assessed against current infrastructure.

It should be noted that this body of work is derived from an overall study_of implementing a HAPS communications architecture in a military context [7]. We refer the reader to that thesis for a complete coverage of the concepts and implementations.

\section{Current Systems Landscape}

\section{Current Military Systems}

The current UK military infrastructure is comprised of three key segments: BOWMAN considers the tactical ground segment, operated by individuals and units. Falcon manages the medium-range terrestrial communications and is the link between BOWMAN and Skynet 5. Skynet 5 is the current geostationary satellite constellation and manages the SATCOM services to the UK military (Figure 2). 


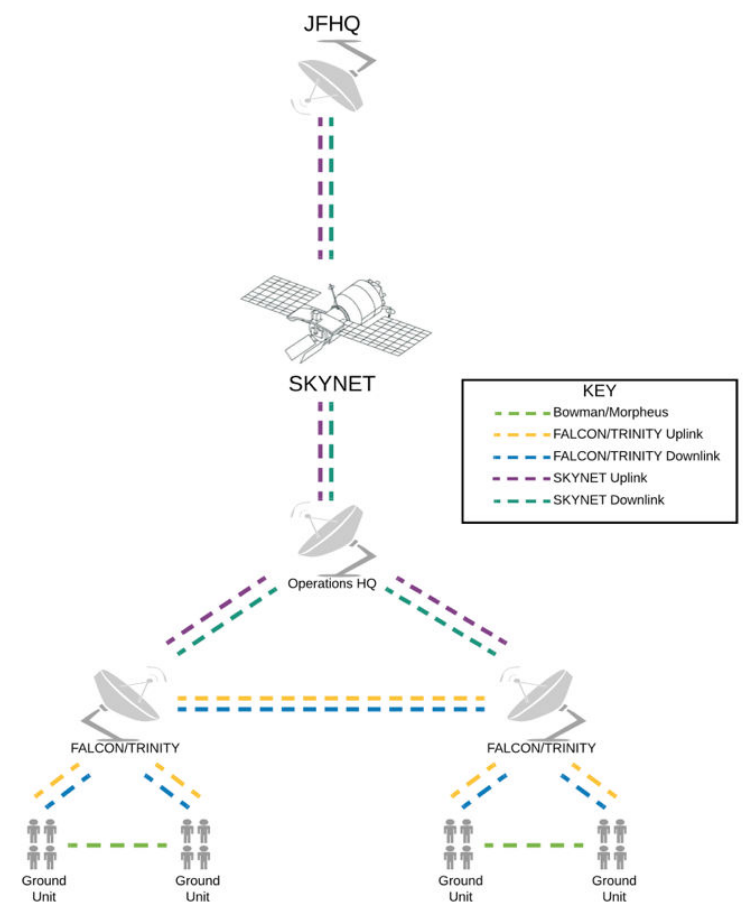

Figure 2: Overview of UK military CIS landscape [8]

\section{Zephyr}

High-Altitude Pseudo-Satellites deviate from conventional unmanned systems as they function at altitudes usually exceeding $20 \mathrm{~km}$. By functioning within the stratosphere, the platforms can negate much of the problems faced by vehicles at lower altitudes. This enables various technologies such as solar power to be employed, resulting in a more environmentally friendly and higher endurance platform upon which a network can be built. The enduring nature of HAPS means that conventional lack of persistence of Air Power is somewhat overcome, enabling airspace to be occupied for an increased (although still limited) period of time.

Airbus' Zephyr S platform has been able to sustain a world record flight duration of almost 26 days. The lightweight structure of a solar powered aircraft means that the payloads are vastly limited, in particular, the Zephyr S allows for up to $5 \mathrm{~kg}$ to be carried by the platform [9]. This major constraint on payload mass could potentially affect overall networking capability and the correction selection of payload is paramount to producing an effective HAPS Service Package.

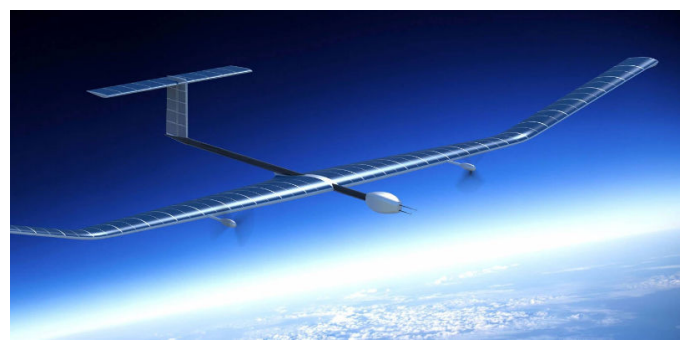

Figure 3: Airbus' Zephyr $\mathrm{S}$ is the chosen platform for this study [9]

Zephyr In the Current System

Zephyr has many potential applications within the current communications infrastructure, it may not be able to fully replace existing systems, however it can be used as a localized network of enhanced performance. Multiple aircraft may be deployed over a specific region whereupon each has the ability to communicate with a GT at a given location. This results in a network that can be rapidly scaled according to operational needs faster than land vehicles. Alongside regional coverage of specific regions, each Zephyr aircraft can operate as a singular L2 node. 


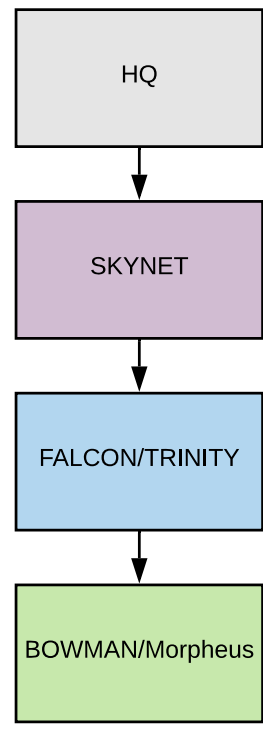

0. Current Method

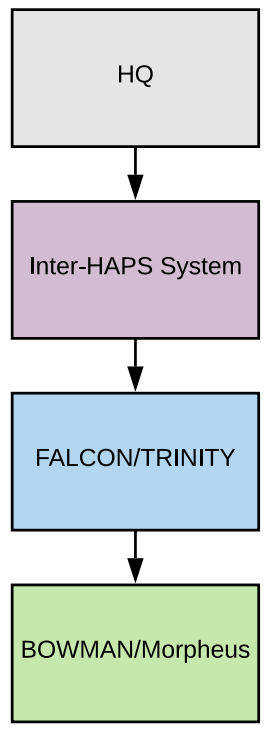

1. HAPS Replace SKYNET

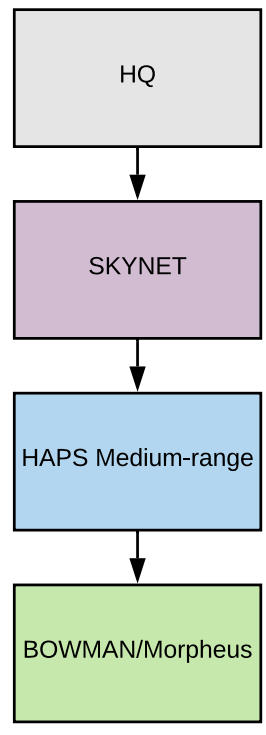

2. HAPS Replace FALCON/TRINITY

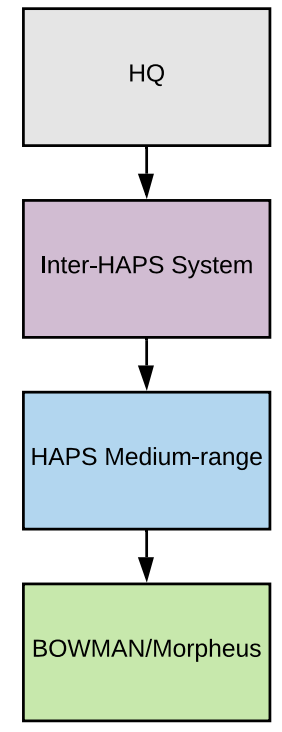

3. Full HAPS

Replacement

Figure 4: Potential services that HAPS could undertake

\section{HAPS Service Package}

As previously mentioned, the investigation does not look to study technologies like-for-like, but rather whole services are analyzed. In order to achieve this, a fleet of HAPS will produce a network constellation that will fulfill a range of services for communications. The current intended application of the HAPS service package will be to replace the steerable spot beams offered by Skynet, as well as enabling long range beyond line of site (BLOS) communications currently unavailable to Falcon and BOWMAN. Once the service package has been produced, a concept of operations will be required, choosing a potential conflict zone that may require the rapid deployment of communications infrastructure.

\section{Network Architecture Overview}

In order to create a baseline for $\mathrm{C} 2$, a baseline network architecture has been defined. Once a foundation for a network had been outlined, the $\mathrm{C} 2$ can be implemented in such a way that the size, design and topology of the network are upheld at all times.

The network architecture consists of a fleet of HAPS, with each aircraft acts as an L2 node, setting up a persistent mesh network. This network will cover a variety of ground stations over the operational area, allowing for beyond line of sight communications across the battlespace. In order to reduce latency times, the network will used standard internet protocol to route the data transmission across the network in the fastest and most reliable route. The number of ground stations will be dependent on the operational needs and will be scaled accordingly, as the network itself is scaled, so will the number of maintenance stations.

The network architecture consists of two systems, each with a unique function. The first has been named the "Ground Terminal to HAPS Link (GT-HAPS Link). This system consists of a downlink beam from the HAPS, providing coverage over a region of $75 \mathrm{~km}$ radius, offering connectivity via a simple bent pipe architecture. The second system has been coined the "Inter-Platform Link" (IPL). The IPL is the connection between each HAPS, producing an interconnected Aerial Network Layer (ANL), vastly increasing the distance over which coverage is produced. 


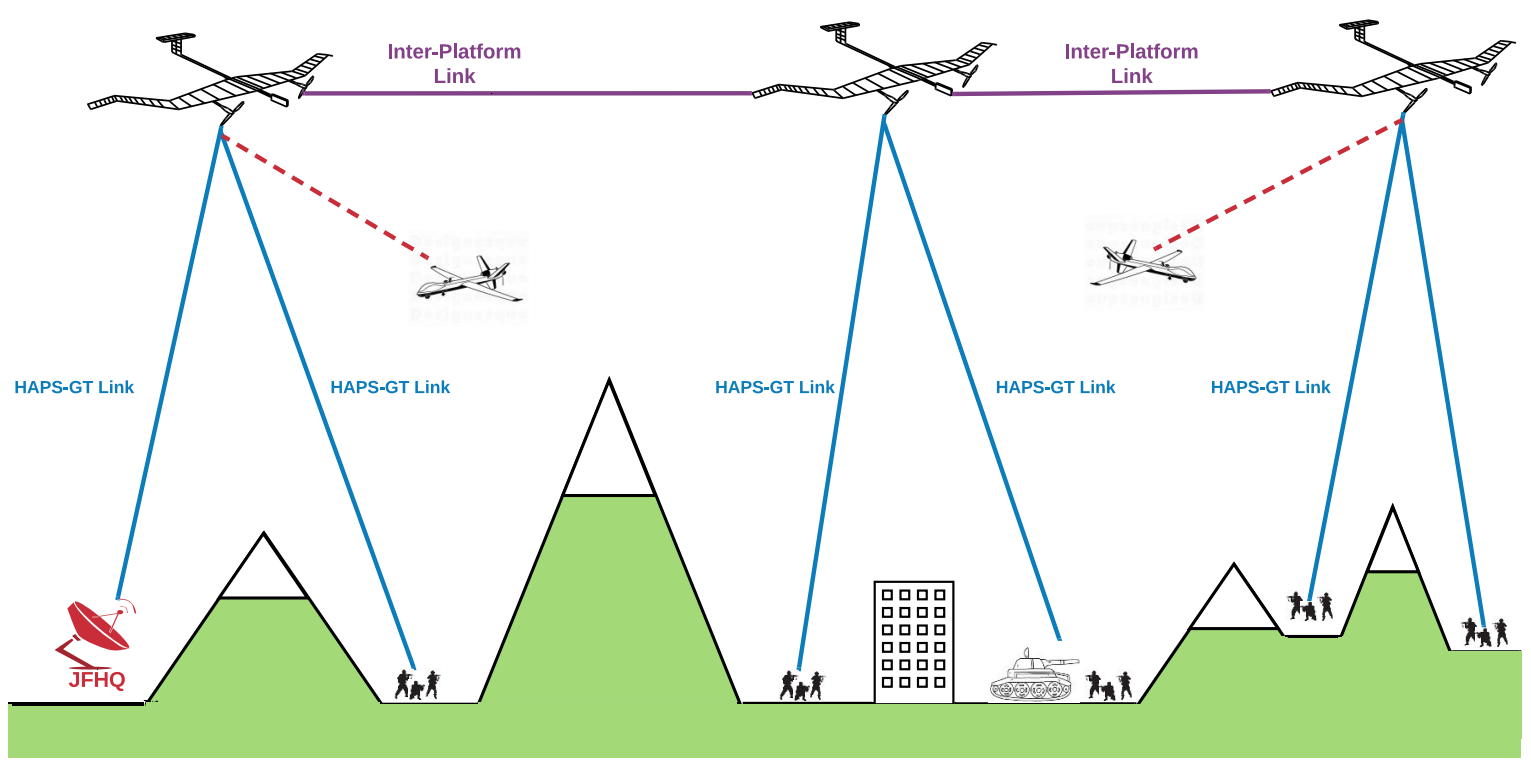

Figure 5: HAPS Network overall architecture design [7]

\section{Payload}

Much like architecture, the payload exhibits influence on the $\mathrm{C} 2$ requirements. The minimum RSS requirements of the HAPS-GT Link and IPL antenna result in the requirement that the HAPS are distanced at $150 \mathrm{~km}$ from one another, with each HAPS providing coverage over a circle of diameter of $150 \mathrm{~km}$. The payload consists of the GetSat Nano for HAPS-GT Link and the combination of Ubiquiti Network's Rocket M5 and AMO-5G13 for the IPL. Full information regarding payload architecture and performance can be found in full in supporting thesis [7]

\section{Command and Control}

Aircraft Cycling Strategy

The flight path is best described as a series of phases (Figure 6), where HAPS will occupy different airspace locations and will perform a different function at each. Before returning for maintenance.

Phase 1: HAPS initial take-off after undergoing production/maintenance. The aircraft will manoeuvre to the required location to initiate Phase 2.

Phase 2: In the intermediary position between lift-off and arriving at the required destination, HAPS will act as a link between Phase 3 and the central HQ (i.e. Joint Forces Headquarters, JFHQ). This is the 'Inter- HAPS' link that will provide the network to emulate SKYNET. There could be multiple aircraft that occupy this Step at defined distance intervals, depending on the total network distance and the communication payload capabilities.

Phase 3: When HAPS arrives at the destination, it will perform the medium-range communications operations, acting as the go-between for Operations HQ and ground units.

Phase 4: When returning from the destination HAPS will continue to support the Inter-HAPS network, offering two potential communications streams for reliability purposes.

Phase 5: HAPS returns to maintenance base, where it will undergo numerous inspections/repairs. 


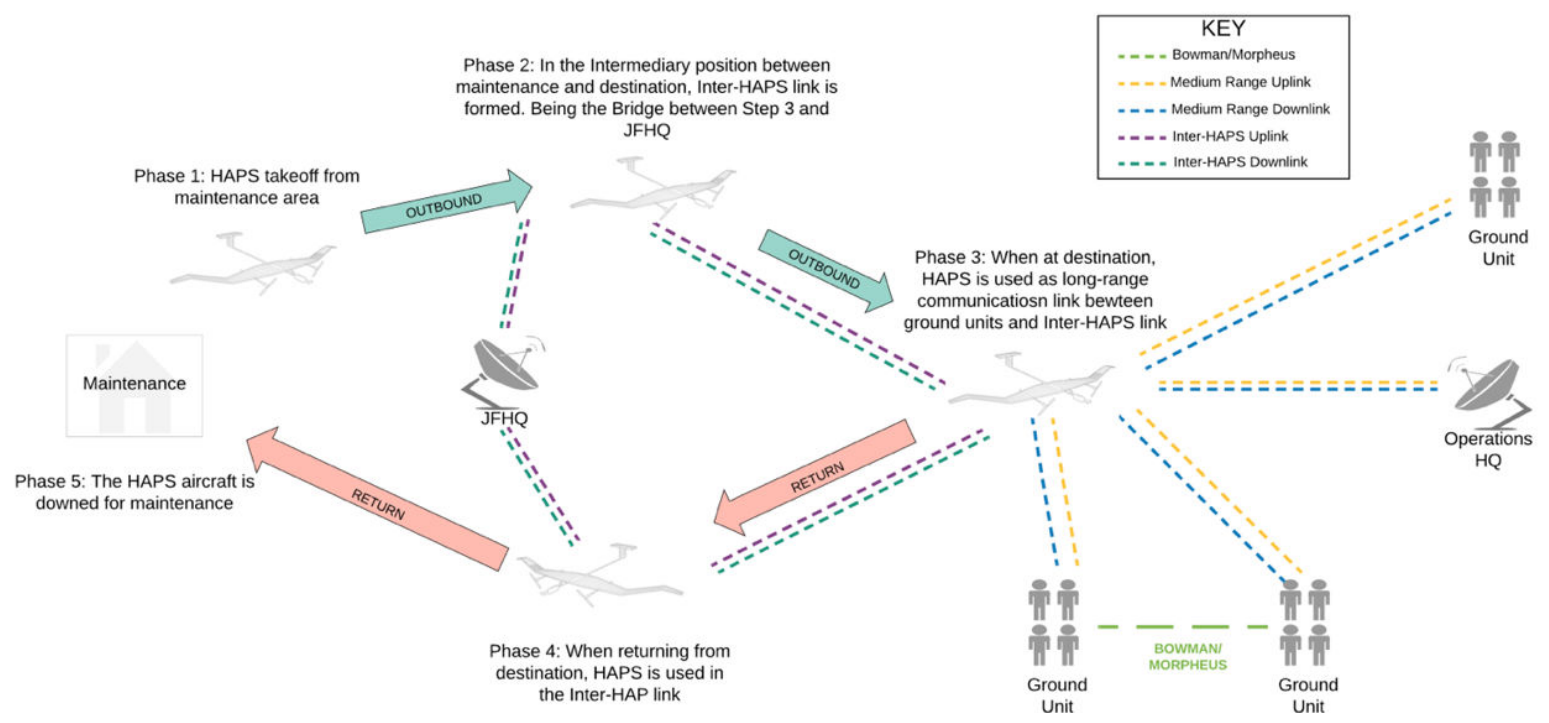

Figure 6: The cycling system that will used to synchronize the HAPS flight path to the network architecture

Combining the flight path of each HAPS with the cycling strategy results in a cyclic trajectory, where each HAPS follows a rotational path across the battlespace, stopping at "Waypoints" to perform HAPS-GT Link duties. The control of the position of each waypoint is the cornerstone to controlling the size and shape of the ANL.

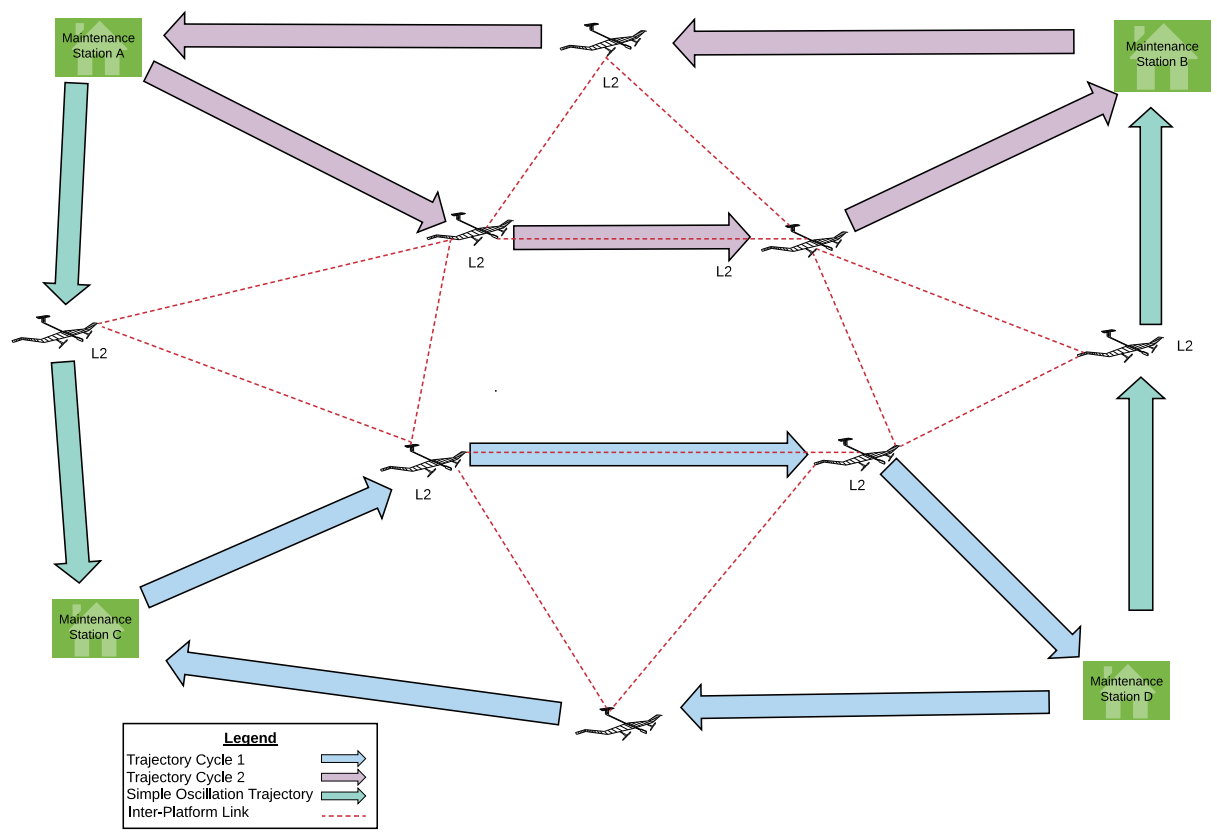

Figure 7: Mesh network set up via the trajectory of multiple HAPS aircraft flying between different maintenance stations

\section{Command and Control Architecture}

In this $\mathrm{C} 2$ architecture, the reporting system functions such that there is a centralized command center that controls the position of each HAPS, however, instead of using a satellite link to send and receive C2 data, the HAPS communications system itself acts as the network to send each command. The HAPS network benefits are realized by making the $\mathrm{C} 2$ self-contained. Firstly, there is no requirement for a satellite uplink, and secondly latency is reduced through use of an ANL. An additional advantage of utilizing this architecture is that extra antenna will not be required for the satellite uplink, resulting in a reduction in payload mass in an already tight $5 \mathrm{~kg}$ constraint. However, there is 
one clear disadvantage to such an architecture. Should one aircraft be broken from the IPL, the ability to control the HAPS will be compromised, resulting in a potential loss of a Zephyr S aircraft alongside its payload. Although this issue is somewhat mitigated by having predefined flight paths, the risk could be further mitigated by introducing an emergency satellite backhaul link to the command station.

\section{Class of Automation}

As the Zephyr S in an unmanned vehicle and the fleet consists of multiple vehicles airborne, it is necessary for a relatively high degree of automation. Using the three criteria for automation [10], a class of automation has been identified: 1) What is the quality of decisions made by the human/machine? 2)What are the ethical and legal requirements for making decisions? 3)What is the accessible information available to the human/machine?

Using the above criteria, there are two principles of automation for the two levels of the system. The first level is the overall shape and position of the HAPS network. As the deviations of position are large and infrequent, a Class B semi-automated system of 'Management-by-consent' has been identified as the having the greatest benefit. This means that the HAPS network reports the position of each HAPS node to the centralized command station, where a human operator will approve the execution of a recommended change of network shape or position.

The second level of automation is the position of each HAPS over the ground units. The HAPS node receives the signal strength for both the HAPS-GT link and the IPL, maneuvering itself to maximize the strength of both. As the maneuvers are small and often, a Class $\mathrm{C}$ automation has been deemed optimum, where each HAPS maneuvers itself automatically according to necessity without the need for input from a human operator.

Table 1: The automation criteria given to the two $\mathrm{C} 2$ systems

\begin{tabular}{|c|c|c|}
\hline System & Automation Class & Description \\
\hline $\begin{array}{l}\text { HAPS Positioning } \\
\text { within Network }\end{array}$ & Class B & $\begin{array}{l}\text { The position of waypoints within the HAPS network is } \\
\text { managed by a human operator. Position is validated by } \\
\text { the reporting system, approved by operator, then } \\
\text { executed by the HAPS }\end{array}$ \\
\hline $\begin{array}{l}\text { HAPS Position } \\
\text { around the } \\
\text { Waypoint }\end{array}$ & Class $\mathrm{C}$ & $\begin{array}{l}\text { The RSS of the HAPS-GT link and IPL is monitored and } \\
\text { each HAPS maneuvers to maximize the efficiency of the } \\
\text { link. Can be overruled by human operator }\end{array}$ \\
\hline
\end{tabular}

HAPS Position within Network: Net Movement of Waypoints

The simplest of the two $\mathrm{C} 2$ strategies is to maintain the shape of the overall network but move the geographical position of the waypoints. In doing so there is a net movement of the HAPS network in the given direction, without changing the relative bearing of each position from each other. The flight duration then updates to accommodate for this drift, temporarily altering the time spent at each destination to account for the migration of the HAPS.

\section{HAPS Position within Network: Network Shape Management}

The network shape is managed by controlling the position of waypoints within the trajectory. This is controlled by a human operator, who inputs the new position of a waypoint, which is then validated by a feedback reporting system. As it is a Class B automation, the $\mathrm{C} 2$ of shape management is dictated mainly by a human operator, with a reporting system that recommends the optimum position of surrounding nodes to maximize efficiency and prevent breakages in links.

The $\mathrm{C} 2$ is outlined as in Figure 8 with the human operator inputting the position of the new waypoint. The reporting system then determines whether there are breakages in links (i.e. IPL distance $>150 \mathrm{~km}$ ) or ground units left not covered. In the event that there is loss of coverage, the reporting system recommends that another HAPS move as well. Once a position is found for each HAPS, the system recommends this to the operator, who then has an option to cancel, override, or confirm the recommendation. 


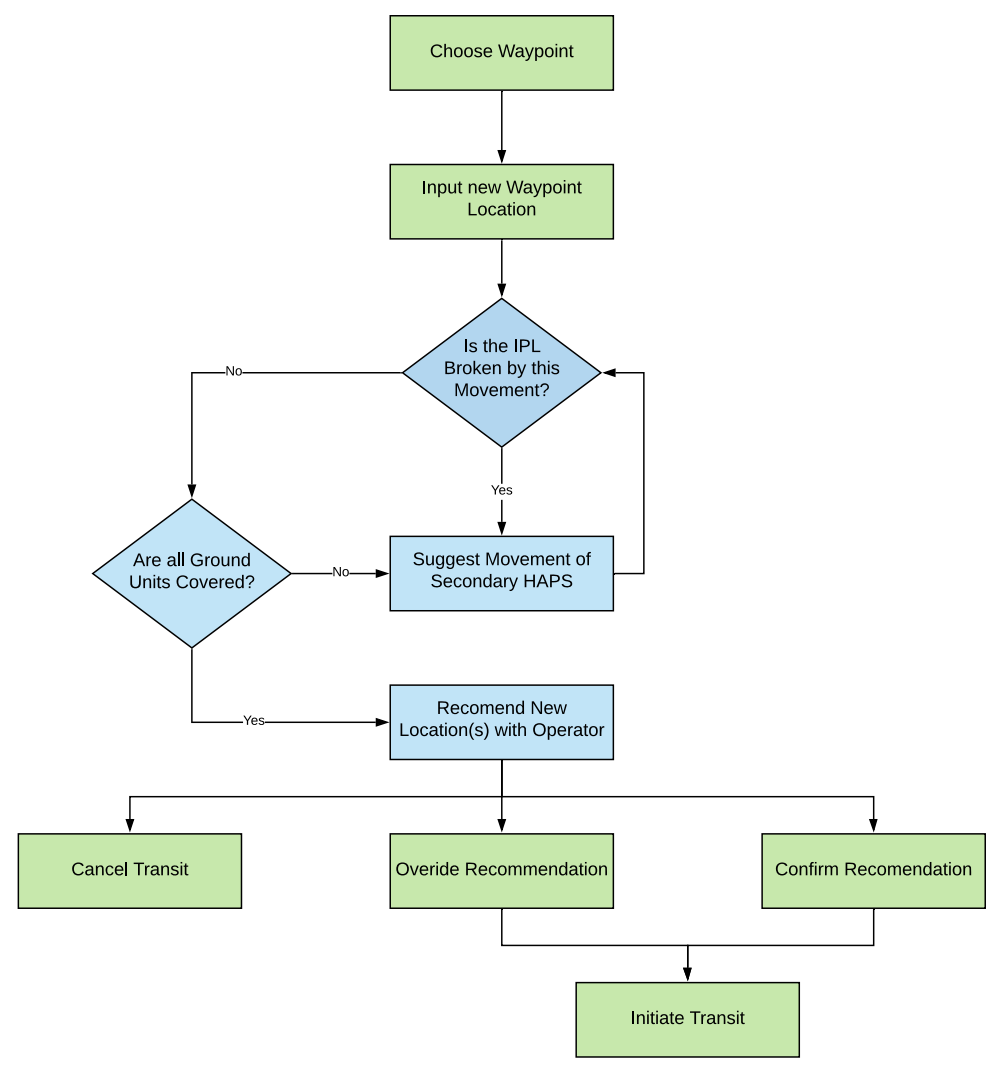

Figure 8: Feedback loop decision process to dictate the shape of the network, based on the OODA decision loop

Utilizing the same shape as in previous examples, the shape may be changed by moving one of the waypoints north to account for the movement of units. This leaves a gap in coverage in the middle of the battlespace, while also severing a link to the IPL. The reporting system identifies this, and recommends that a nearby HAPS transits alongside it, allowing for coverage to be maintained, while also upholding the IPL (Figure 9).
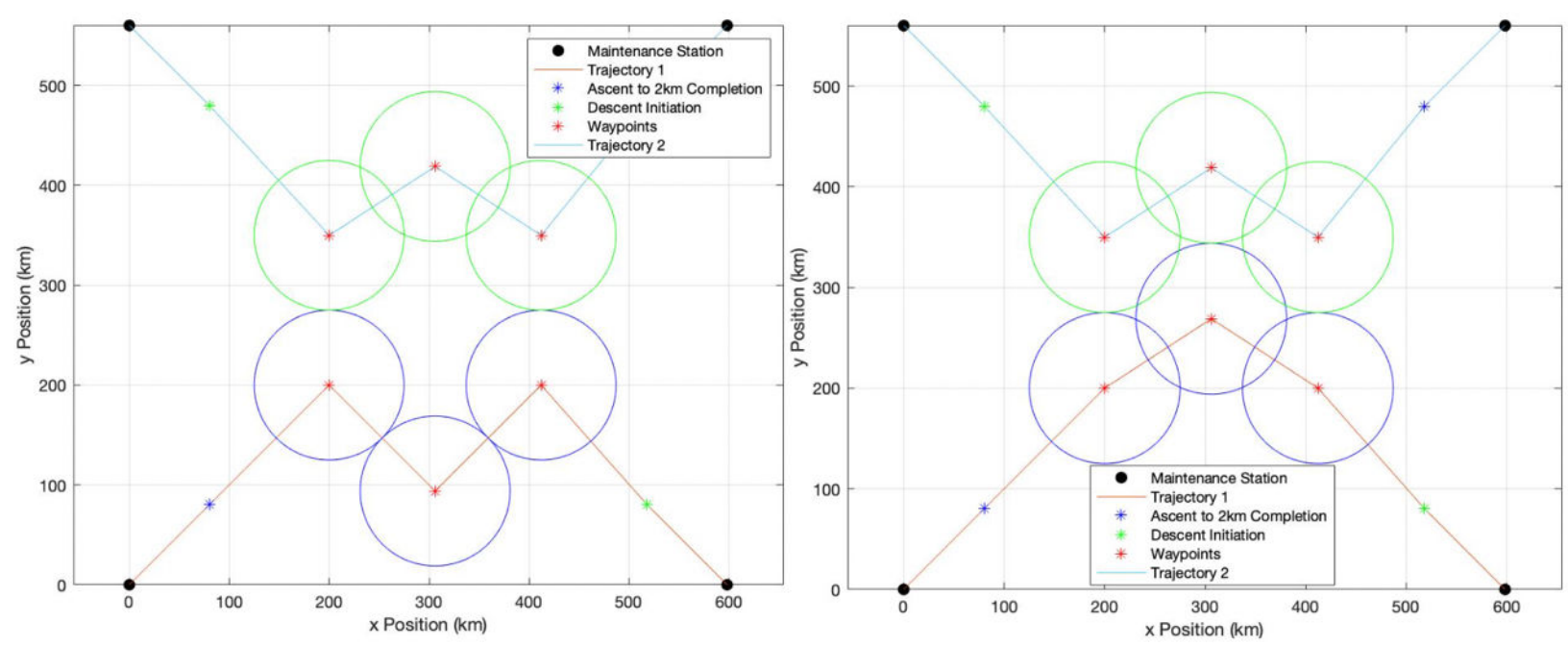

Figure 9: (Left) The change in position dictated by the operator. (Right) The new position of HAPS recommended by the reporting system, making sure the appropriate IPL and coverage area (circles) are upheld 
HAPS Position Around Waypoints

The second level of C2 of the HAPS network is more localized. In order to maximize the RSS of the surrounding units, each HAPS node can be automated with a Class $\mathrm{C}$ architecture to maximize communications with surrounding forces. In order to achieve this, the HAPS maneuvers itself into a position that enables the greatest RSS from both the HAPS-GT Link and the IPL. Each HAPS monitors the RSS from surrounding beams, then determines the best position to optimize the link strength. It does this by identifying the weakest RSS, and positions itself closer to the unit, while also monitoring the drop in RSS from other links. When the weakest strength becomes equal to one of the signal strengths, the HAPS remains at this location, until another small deviation is deemed necessary.

As the IPL has a limited capacity, a threshold RSS of $73 \mathrm{dBm}^{5}$ is required to be upheld. If the movement of the HAPS is predicted to be outside of this received power, the HAPS moves to within 5dBm of the RSS threshold. The IPL RSS is prioritized at all times, with the main deviations of the HAPS around the waypoint undertaken in order to improve this link.

\section{Ground Terminals and Handover}

The HAPS access nodes in the ANL are mobile, requiring a trajectory that passes over multiple units for finite periods of time. As a result, there is a requirement that a comprehensive handover strategy is put in place to allow the seamless transition of communications from one Zephyr S to another. The handover process is considered to be an extension of $\mathrm{C} 2$ and is a Class 4 fully automated system. This section will explore the handover initiation and process and how it is tied to the overall $\mathrm{C} 2$ of the network.

\section{Handover Initiation}

The handover initiation is the process in which the GT signals that there is a preferable HAPS beam that is better suited to undertake the HAPS-GT link, than that which it is currently using. The optimal link is assessed by comparing the RSS from the beam it is communicating with (Server), with other surrounding beams (Candidate), using a set of predefined parameters to prevent a drop in calls or QoS. Utilizing the RSS as the main measurable for handover has the benefit for determining the optimal link for the system as it is directly related to the performance of the network. There are multiple hazards that can impact the QoS that require mitigation. Fluctuations in RSS caused by atmospheric attenuation and relative movement of the HAPS cause the handover to be constantly initiated between a Server and Candidate HAPS. This 'Ping Pong' effect is a common phenomenon that results in the deterioration of link performance, due to unnecessarily increases the load on the system as communication bounces to and from different HAPS beams.

In order to maximize network capacity, handover rates are minimized to reduce network load [11] by including a threshold frequency that a server beam is required to be above, in order to begin a initiate a handover. The threshold RSS is determined by the when the RSS is above $-97.31 \mathrm{dm}$, this correlates to the GT being at $17^{\circ}$ to the candidate HAPS. This means that the handover will not be initiated until the unit is well within the coverage zone $(6.81 \mathrm{~km})$, resulting in the ping pong effect being significantly reduced at the border between HAPS coverage areas.

In addition to a threshold frequency, a hysteresis margin is implemented in order to further reduce the Ping Pong effect. The hysteresis margin is inversely proportional to handover rate, however, a larger margin results in a greater handover delay, meaning that a trade-off needs to be made between handover rate and handover delay. When determining the hysteresis margin, an added factor of HAPS velocity dictates the number of handovers that may occur. Investigations into this phenomenon [12] found that beyond $6 \mathrm{~dB}$ there was little variation in the number of handovers for the range of velocities. In the context of the HAPS network this margin is deemed optimal, as there are there will be a large variation of velocities of ground units, as well as variations in velocities of surrounding HAPS. This one size fits all parameter means that there is an appropriate handover threshold that has the same effect on all units regardless of their velocities, allowing for a consistent and robust handover threshold strategy.

\section{Handover Initiation Process}

The handover initiation process (Figure 10) follows a simple hierarchal structure such that it initially only measures and compares the RSS of the incoming beams. The process only proceeds to the next stage if the Candidate Beam has a greater RSS than the current Server Beam. By implementing this initial condition, the ping pong effect can be negated at the beginning of the loop, as the Server is already preferred when compared to the Candidate, preventing

${ }^{5} 73 \mathrm{dBm}$ represents the minimum RSS the chosen payload is required to enable the maximum capacity of 54Mbps 
unnecessary handover. The next condition is such that the Server beam is above the required threshold and hysteresis margin. In the event that it does not exceed this value, the process is once again halted, preventing any handover that may jump between the two potential HAPS links.

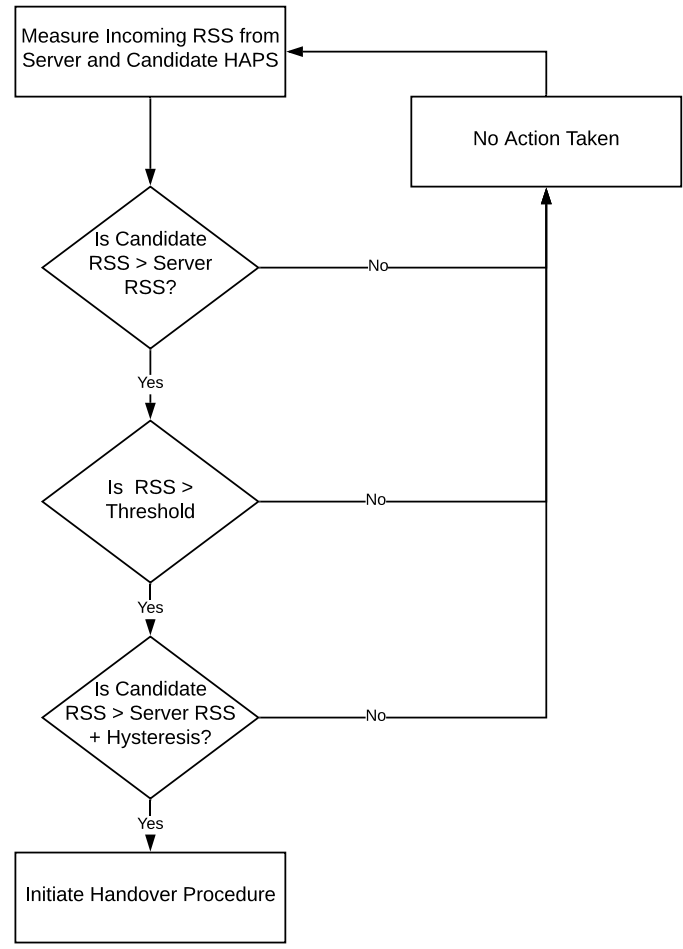

Figure 10: Handover initiation process that initially filters out weaker Candidate beams, then applies the threshold and hysteresis conditions to prevent the ping pong effect.

In order to verify the handover initiation strategy, a simulation was performed. This consisted of two aircraft flying over a ground terminal and a series of handovers being initiated. Utilizing RSS alone produced 17 handover initiations, whereas RSS with a threshold and hysteresis parameter yielded only 1 handover [7]. This reduction drastically reduces the network load, reducing the amount of drop calls and loss of connectivity.

\section{V.Concept of Operations Scenario}

In order to measure the capabilities of the HAPS Network against the current communications infrastructure, a Concept of Operations (ConOps) has been formulated. This ConOps scenario looks to investigate a specific situation where both HAPS and standard CIS architecture are deployed, a theoretical performance analysis of each infrastructure is then undertaken. Once achieved, the communications packages in their entirety are compared, rather than a like-for-like study of technology.

\section{Context}

A case study of operations situated in Southern Iran has been undertaken ${ }^{6}$, where communications are required to be deployed over an area of diameter $\sim 500 \mathrm{~km}$. In this scenario, HAPS are required to be deployed in an emergency due to the capabilities of Skynet's Spot beam becoming compromised due to insurgent disruption to communications. A theoretical Communications Service Requirement [13] has been formulated to provide a foundation upon which the infrastructure is built around.

\footnotetext{
${ }^{6}$ Southern Iran has a latitude of $\sim 30^{\circ} N$, which is within the latitude limits of Zephyr S. This study has used this location based purely on potential operational zones that are within the latitude constraints of Zephyr S.
} 
Table 2: Communications Service Requirement for ConOps case study

\begin{tabular}{|c|c|c|c|}
\hline Service & Classification & Solution & Users \\
\hline \multicolumn{4}{|c|}{ DATA } \\
\hline $\mathrm{C} 2$ & Top Secret & IPL/Skynet & 10 \\
\hline $\mathrm{C} 2$ & Mission Secret & HAPS-GT/Skynet & 50 \\
\hline ISR & Top Secret & IPL/Skynet & 5 \\
\hline \multicolumn{4}{|c|}{ VOICE } \\
\hline VOIP (UK) & Top Secret & IPL/Skynet & 10 \\
\hline VOIP (UK) & Mission Secret & HAPS-GT/Skynet & 50 \\
\hline VOIP (NATO) & Mission Secret & HAPS-GT/Skynet & 50 \\
\hline VOIP & Classified & Falcon & 50 \\
\hline VOIP & Mission Secret & Bowman & 100 \\
\hline \multicolumn{4}{|c|}{ Messaging } \\
\hline $\mathrm{C} 2$ & Top Secret & IPL/Skynet & 10 \\
\hline $\mathrm{C} 2$ & Mission Secret & HAPS-GT/Skynet & 50 \\
\hline Other & Unclassified & Falcon & 100 \\
\hline
\end{tabular}

\section{Methodology}

The case study is undertaken by producing a communications infrastructure for the standard military communications strategy (Baseline), followed by building the HAPS Network, which will fulfil the needs set out by the Communication Services Requirement. Once produced, analysis of the performance of the two systems is then carried out by using a series of qualitative parameters (Table 3 ) developed from the NEC military aspirations [1]. In addition to this, the two systems are also compared by analyzing performance of each network via quantitative parameters (Table 4).

It should be noted that when determining the responsiveness of communication systems, there are two criteria that UK MoD CIS doctrine [13] determines as phases of deployment. The first of which is the time taken for initial operational capabilities to commence, in this case study, this is regarded as the moment the first node becomes available for communications. The second criteria is when the communications network becomes fully operational, with all nodes in the correct point.

Table 3: Qualitative measurables that are used to analyze the performance of each infrastructure. Each measurable is developed from the NEC 'Military Aspirations' [1] for communication systems development

\begin{tabular}{|c|c|}
\hline Attribute & Description \\
\hline Responsive & $\begin{array}{l}\text { The ability for a HAPS to be deployed rapidly to meet the operational needs will be monitored. It is } \\
\text { hypothesized that it will benefit extremely as a platform that will be able to provide a short-term network } \\
\text { to either replace downed systems, or first contact deployment of forces. }\end{array}$ \\
\hline Robust & $\begin{array}{l}\text { Although more a qualitative measurable, the ability for the service package to maintain secure } \\
\text { communications in the advent of an aircraft/payload disability will be analyzed. This will be done by } \\
\text { subtracting a node from the network and observing if communications lines are broken and how the HAPS } \\
\text { can reposition to maintain communication. }\end{array}$ \\
\hline Broad & $\begin{array}{l}\text { Another qualitative measurable, the various regions that HAPS can operate over will be identified and } \\
\text { compared to both satellite and terrestrial communications. }\end{array}$ \\
\hline Flexible & $\begin{array}{l}\text { The flexibility of the network will be measured by its ability to route the data through multiple nodes } \\
\text { unhindered with minimum latency. }\end{array}$ \\
\hline Adaptable & $\begin{array}{l}\text { This will also need to take into account the ability for the HAPS to migrate effectively according to traffic } \\
\text { demands, making sure that adequate time is also left to allow the aircraft to return for maintenance. }\end{array}$ \\
\hline Scalable & $\begin{array}{l}\text { It is assumed that the HAPS network will enable rapid deployment over localized regions, however, in } \\
\text { order to compete with current technologies, the ability for the network to be scaled according to operational } \\
\text { needs will be analyzed. This may involve adding/subtracting aircraft, or conversely increase/decrease the } \\
\text { spacing between different HAPS }\end{array}$ \\
\hline Interoperable & $\begin{array}{l}\text { Identified as a key requirement, HAPS will be measured on its ability to integrate with other military } \\
\text { communication systems. }\end{array}$ \\
\hline Reactive & $\begin{array}{l}\text { This measurable will monitor the time taken for the network to be deployed. An estimated time for initial } \\
\text { and full capacity will be made and compared to its terrestrial/space segment counterpart. }\end{array}$ \\
\hline Cost Effective & $\begin{array}{l}\text { A cost analysis will be undertaken on all systems, allowing for a comparison between architectures as to } \\
\text { which is the best value for money. }\end{array}$ \\
\hline
\end{tabular}


Table 4: Network performance parameters that are used to assess the two communications infrastructures

\begin{tabular}{|c|c|}
\hline Attribute & Description \\
\hline Data Rate & $\begin{array}{l}\text { The maximum and assured data rate for the system will be estimated and be compared to that of } \\
\text { current infrastructure. }\end{array}$ \\
\hline Bandwidth & $\begin{array}{l}\text { The required bandwidth to maintain communications will be stated and compared to that of } \\
\text { Skynet/Falcon }\end{array}$ \\
\hline Latency & An estimation of latency times will be made for initial and optimum conditions \\
\hline Channels Per Node & $\begin{array}{l}\text { The ability for HAPS to maintain multiple connections is crucial to producing a mesh network. The } \\
\text { capability for HAPS to open up several channels to different nodes will be evaluated. }\end{array}$ \\
\hline Total Coverage Area & $\begin{array}{l}\text { In order to compete with Regional and Spot beams produced by Skynet, HAPS will have to cover a } \\
\text { minimum area. The total coverage area allowed by the network will be measured and subsequently } \\
\text { compared to that of Skynet. }\end{array}$ \\
\hline
\end{tabular}

\section{Baseline Scenario}

The baseline scenario consists of the standard communications infrastructure that is currently used by the UK military. The communications network consists of the Bowman Tactical ground communications network, the Falcon medium range communications network and finally Skynet 5's Spot beam SATCOM services (Table 5).

Table 5: Skynet 5 beam specifications

\begin{tabular}{|l|l|l|l|l|l|}
\hline \multicolumn{7}{|c|}{ Skynet 5 Beam Specifications } \\
\hline Element & Units & Global Beam & Wide Beam & Regional Beam & Spot/Theatre Beam \\
\hline Max No. of Beams & - & 2 & 3 & 6 & 9 \\
\hline Peak Directivity & $\mathrm{dBi}$ & - & 22 & 25 & 30 \\
\hline Coverage Area & $\mathrm{km}$ & - & 1750 & 1250 & 600 \\
\hline EIRP & $\mathrm{dBw}$ & 41 & 41 & 41 & 50.5 \\
\hline Edge of Cover G/T & $\mathrm{dB} / \mathrm{k}$ & - & -10.2 & -0.5 & 4.5 \\
\hline Data Rate (Uplink) & $\mathrm{Mbps}$ & 1 & - & 2 & 14 \\
\hline Data Rate (Downlink) & $\mathrm{Mbps}$ & 2 & - & 4 & 14 \\
\hline $\begin{array}{l}\text { Antenna Support } \\
\text { >1Mbps }\end{array}$ & $\mathrm{m}$ & 1.2 & - & - & 0.45 \\
\hline
\end{tabular}

Starting at points stationed around the battlefield (positions analogous to HAPS Network 'Maintenance Stations', Skynet and Falcon are deployed using the Mowag Duro III and HX60 vehicles respectively. The Reacher Terminal is mounted on a Mowag Duro III which has a top speed of $100 \mathrm{~km} / \mathrm{h}$, Falcon is mounted on a HX60 which has a top speed of $88 \mathrm{~km} / \mathrm{h}$, however uneven terrain and dusty roads limit the vehicles to $30 \mathrm{~km} / \mathrm{h}$, vastly increasing the deployment rate of both systems. It is assumed that the Bowman system is deployed alongside units, which can be carried in by air vehicles, resulting in a deployment rate under an hour.

Once full capabilities are running, Skynet and Falcon provide speeds $14 \mathrm{Mbps}$ and $8 \mathrm{Mbps}$, with latencies due to propagation and processing delays resulting in $260 \mathrm{~ms}$ and $20.27 \mathrm{~ms}$ respectively.

Table 6: Network deployment and performance results for the standard SATCOM communications providing coverage over a $500 \mathrm{~km}$ area

\begin{tabular}{|l|l|l|l|l|l|l|}
\hline Element & Range (km) & $\begin{array}{l}\text { Single User } \\
\text { Data Rate }\end{array}$ & $\begin{array}{l}\text { Estimated } \\
\text { Latency } \\
\text { (ms) }\end{array}$ & $\begin{array}{l}\text { Time Until } \\
\text { Initial } \\
\text { Performance } \\
\text { (hrs) }\end{array}$ & $\begin{array}{l}\text { Time Taken } \\
\text { for Performance } \\
\text { Perogram } \\
\text { (hrs) }\end{array}$ & $\begin{array}{l}\text { Full } \\
\text { Cost (£bn) }\end{array}$ \\
\hline Bowman & $15 \mathrm{~km}$ & 0.54 & - & $<1$ & $<1$ & 1.90 \\
\hline Falcon & $40 \mathrm{~km}$ & 8.00 & 20.27 & 6.90 & 10.20 & 0.38 \\
\hline Skynet & $500 \mathrm{~km}$ & 14.00 & 260.00 & 6.90 & 10.20 & 3.66 \\
\hline
\end{tabular}

Skynet's Spot beams are steerable and can be shaped according to need. This means that SATCOM services can be distributed effectively throughout the battlespace, with the only limitation being the range in which the Mowag Duro III can travel $(\sim 350 \mathrm{~km}[14])$. 


\section{HAPS Network Scenario}

Using the same scenario as previously outlined, where various units stationed across a Battlezone $\sim 500 \mathrm{~km}$ in diameter. The deployed HAPS network consists of two cyclic and two oscillatory trajectories (Figure 11), where each HAPS navigates to a designated waypoint, where it undertakes nominal HAPS-GT Link functions. Once full deployment is achieved, the IPL is initiated, and the HAPS Communication Network is optimized.

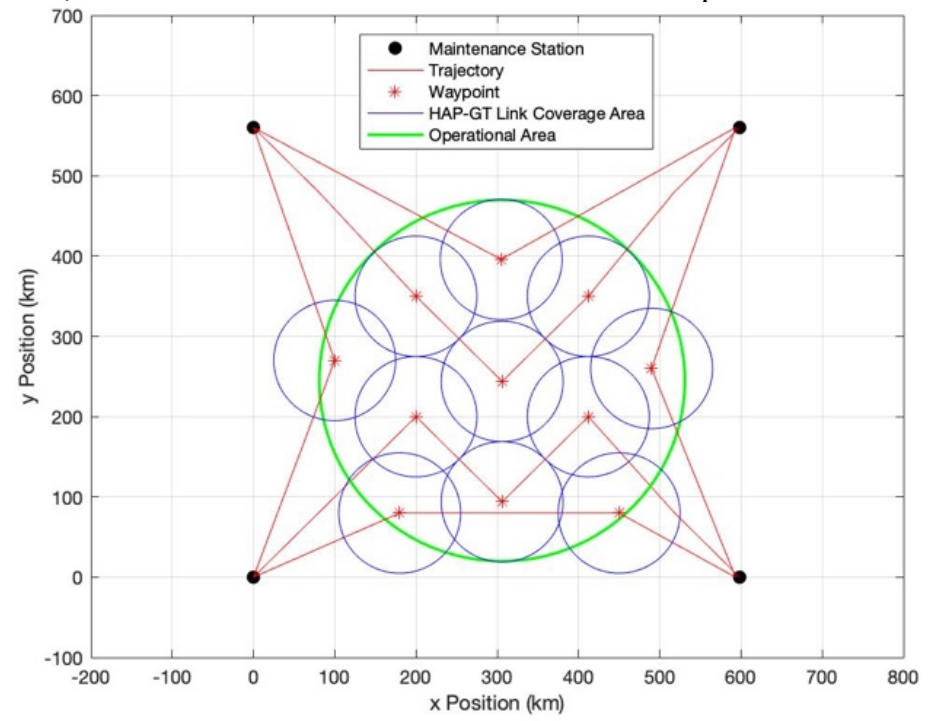

Figure 11: Four trajectories allow for a total of 11 HAPS provide coverage to ground units as well as initiating the IPL

The coverage area of the configuration of HAPS is such that it does not cover the entirety of the designated battlespace (Figure 11)Error! Reference source not found., however, the C2 allows for the HAPS to migrate alongside the movement of units within the battle zone, resulting in a more efficient coverage.

The HAPS Network IPL standard data rate for 50 users spread over 10 channels results in a single user data rate of $1.2 \mathrm{Mbps}$, however, in this ConOps scenario, the Communications Services Requirement dictates that 30 users are needed for $\mathrm{C} 2$ and priority command purposes.

The HAPS Network makes use of the low latency that is provided by an Aerial Network Layer, the lower altitudes result in HAPS-GT Link latency times of $20.51 \mathrm{~ms}$, rivalling Falcon's own latency of $20.27 \mathrm{~ms}$. The IPL latency time detailed in Table 7 represents the latency as a result of the data passing through a maximum of 5 nodes from one side of the battlefield to another. In reality, this may be significantly lower due to the message not needing to pass through as many nodes.

The deployment of the HAPS network is a lot faster ${ }^{7}$ than its medium range terrestrial counterpart, Falcon. The initial deployment phase of the HAPS-GT Link is 4.4hrs. Once deployed, the HAPS-GT Link can undertake the medium range communications of Falcon, at a slight reduction in capacity ${ }^{8}$ until the system is fully deployed. Shortly after initial deployment phase is complete, the full network can be set up within 6.45 hours, whereupon the IPL can then undertake full communications.

\footnotetext{
${ }^{7}$ It should be noted that the lightweight ROK ground terminals are deployed alongside Bowman and Tactical ground units.

${ }^{8}$ When HAPS undertakes the extra user load required by Falcon, the single use capacity drops to 6.5Mbps for 2.5-5.8 hours until Falcon is operational.
} 
Table 7: Network deployment and performance results for the HAPS Communications Network providing coverage over a $500 \mathrm{~km}$ area

\begin{tabular}{|lllllllll|}
\hline Element & $\begin{array}{l}\text { Range } \\
(\mathbf{k m})\end{array}$ & $\begin{array}{l}\text { Single User } \\
\text { Data Rate }\end{array}$ & $\begin{array}{l}\text { Estimated } \\
\text { Latency } \\
(\mathbf{m s})\end{array}$ & $\begin{array}{l}\text { Time Until } \\
\text { Initial } \\
\text { Performance } \\
\text { (hrs) }\end{array}$ & $\begin{array}{l}\text { Time Taken } \\
\text { for Program } \\
\text { Performance } \\
\text { (hrs) }\end{array}$ & $\begin{array}{l}\text { Full } \\
\text { Cost (£bn) }\end{array}$ \\
\hline Bowman & $15 \mathrm{~km}$ & 0.54 & - & $<1$ & $<1$ & 1.90 \\
\hline Falcon & $40 \mathrm{~km}$ & 8.00 & 20.27 & 6.90 & 10.20 & 0.38 \\
\hline HAPS-GT Link & $150 \mathrm{~km}$ & 12.01 & 20.51 & 4.40 & 6.45 & 0.70 \\
\hline IPL & $500 \mathrm{~km}$ & 1.80 & $122.60^{9}$ & 6.45 & 6.45 & "As above" \\
\hline
\end{tabular}

\section{Discussion}

Utilizing Error! Reference source not found. as a cornerstone for analysis, the performance of the HAPS network can be sufficiently analyzed against nominal SATCOM services using MoD-defined parameters. Both networks are can be deployed very quickly (over a matter of hours until full capability), however, Skynet is severely limited by its use of the Reacher ground terminal. The deployment speed of the Mowag Duro III reduces the rate at which SATCOM services can be introduced the battlespace, resulting in a lack of full communications for over 10 hours. The HAPS network takes advantage of the 'Speed' aspect of military Air Power [6], enabling preliminary functionality of the HAPS-GT Link within half the time it takes to deploy all nominal systems. In addition to this, the HAPS-GT link can undertake the duties of Falcon while the system is being deployed (albeit at a slight drop in QoS), allowing for localized communications over an operational area to be initiated. The full capabilities of the HAPS Network (including the IPL) are functional before even the initial capacity of nominal SATCOM services are initiated, making the HAPS network far more responsive to emergency than current architectures.

Both SATCOM and the HAPS Network are robust, however in this aspect, Skynet proves to be the more resilient of the two architectures. Although there are significant threats to SATCOM services from adversaries, each Skynet 5 satellite is equipped with nuclear hardening material, as well as anti-jamming strategy. Using the stratosphere as the operational environment, the Zephyr S mitigates the hazards caused by high windspeeds and cloud cover. However, in order to get to the required altitudes, each HAPS must climb through troposphere, where winds speeds are highly variable. This causes a high degree of concern as the HAPS is at a great risk of failure due to adverse weather conditions. This concern was realized in a recent crash of a Zephyr S test craft while taking off in turbulent conditions [15].

One significant issue with the HAPS network is that it does not cover the entirety of the battlespace as Skynet's Spot beam. This means that of the two, Skynet is already considered providing a broader network coverage. The HAPS Network has a slight advantage over Skynet as although it does not entirely cover the battlefield, the coverage it does give can be concentrated more on the ground forces, preventing unnecessary zones from receiving a downlink beam. This could prove to be crucial for operational security, as it means that coverage can be tailored to provide connectivity to ground forces, while avoiding broadcasting a beam to enemy forces.

The flexibility of Skynet's Spot beam is undeniable. The on-board reactive antenna allows for the size and shape of the coverage to be tailored to the needs of the operation. However as previously stated, the Level 3 automated command and control of the network allows for the shape of the HAPS network to be changed significantly over a short time span. Whether the HAPS network occupies a circular, or aligning in a straight line to provide a narrow column of coverage, the HAPS network gains the advantage over Skynet by enabling an almost unlimited range of choices of network shapes and topologies at a cost to coverage area.

The HAPS network can be scaled according to the needs of the Strategic and Operational level commanders by adding extra HAPS and greater number of waypoints. However, when compared to Skynet services, which can provide nationwide or even global coverage, it is evident that in its current state HAPS cannot compete with the scalability of SATCOM services.

\footnotetext{
${ }^{9}$ Value represents the maximum latency for a signal passing through a total of 5 nodes from one side of the battlespace to the other
} 
The HAPS-GT link utilizes military Ka Band frequencies, enabling it to be pre-emptively optimized for military use. With regards to UK communications infrastructure, which uses X-Band spectrum for nominal communications, equipment used for ground services may have to be altered to accommodate this use. In this case study, interoperability is maintained by allocating GetSat's ROK terminal to units, enabling all users to access the HAPS Network regardless of existing technology.

When considering the cost of the two architectures, it must be taken into account that although the HAPS network is significantly cheaper than Skynet, it does not provide the full functionality afforded by SATCOM services. Not only does Skynet consistently provide greater data rates than the IPL, but it also can replicate it over a global coverage area. The $£ 3.66 \mathrm{bn}$ price tag for the constellation of five satellites is much greater than the estimated $£ 0.65 \mathrm{bn}$ constellation of eleven Zephyr S (plus four added for mitigation). For its cost, the HAPS Network provides a high capacity, localized communications service for a short range (12Mbps over $150 \mathrm{~km}$ for the HAPS-GT Link). The network can then provide added range through the IPL at a significant decrease in capacity. When determining the cost effectiveness of this architecture, the function of the HAPS network as a rapidly deployable network in the event of an emergency must be noted. In the event that SATCOM services are compromised, a fleet of HAPS that can be dispatched, providing connectivity to stranded units. When considering how HAPS can be harnessed in an emergency, the £0.65bn investment into a fleet of Zephyr S could potentially be the key to operational success where otherwise a lack of communication (and therefore a lack of battlefield coordination) may have caused a significant loss of life.

\section{Conclusions and Future Work}

When compared to standard SATCOM services, HAPS offers a cost effective and rapidly deployable communications network that can be harnessed in an emergency situation. In addition to mitigating compromised satellite services, the HAPS network also offers a redundancy strategy to the medium-range terrestrial communications offered by Falcon. Although significant gains in value for money and response times are expected, the proposed HAPS network shows a significant lack of functionality when compared to Skynet. The HAPS Network enables more localised services, whereas the constellation of geosynchronous satellites allows for global communications to be undertaken. In addition to this, the limitations of the Zephyr S vehicle are such that it can only operate in a narrow corridor of $\pm 40^{\circ}$ latitude, meaning its applications are severely limited in scope.

The command and control of a fleet of HAPS produces a wide variety of challenges, especially when automating each aircraft to a certain degree. A predefined flight plan for each HAPS enables each aircraft to have a predictable trajectory that can be tracked by a centralised command centre. By controlling the HAPS via altering the waypoints at which each HAPS stays, the fleet position and shape can be dictated by the operator, enabling a flexible and responsive. By exacting a flight duration monitoring strategy, each Zephyr S aircraft can monitor how long it is spending at each waypoint, preventing it from exceeding the 26-day limit, resulting in a command strategy that is synchronised to the flight capabilities of the platform.

By initiating a C2 system that uses the HAPS network itself to send and receive $\mathrm{C} 2$ commands, necessity for a backhaul satellite link is reduced. This means that it can be used in the event that SATCOM services (such as Skynet) are compromised. The command strategy does still rely on satellite systems somewhat, utilising GNSS services for positioning and timing. However, with many commercial satellites available to provide this service, the HAPS can remain independent of Skynet, while still being able to monitor ephemeris data. There are a variety of weaknesses when relying on the HAPS network to command the fleet; the primary of which being, in the event of multiple links becoming compromised, the command of a large portion of the fleet could be lost. This hazard is overcome somewhat by predetermining the flight paths of the HAPS, meaning that in the event of a mass loss of communications with HAPS, the aircraft will continue on their nominal flight path until they land.

The handover process allows for the GT to transition between communications with multiple HAPS as they fly overhead. The outlined initiation process results in an appropriate number of handovers to be undertaken while also mitigating detrimental effects such as the aforementioned ping pong effect. This study has utilised measuring the RSS against various defined parameters, allowing for the optimal signal to be chosen, as well as reducing the number of handovers.

It is apparent that further research into a self-sustaining HAPS network needs to be undertaken. By implementing a C2 strategy that enables the full automation of a fleet of HAPS, with minimal satellite backhaul link will result in an alternative communications strategy to current methods. As such, the handover initiation process may be improved 
further by using predictive algorithms and navigation filters to use the HAPS movement and position to predict optimum handover. In this study, it has been deemed unnecessary, as the number of handovers that will take place are small relative to a cellular network, where multiple handovers are required over a short duration. However, as fleet size and complexity increases, a more robust system of handover may be required.

\section{References}

[1] DCDS (2003) Network Enabled Capability - Concpet Outline.

[2] Gorove, S. (1967) The Outer Space Treaty, Bulletin of the Atomic Scientists. doi: 10.1080/00963402.1967.11455151.

[3] Brig Gen L. F. Kwiatkowski, M. J. Daugherty, Lt Col. C. 0. Cornell, M. A. K. and P. B. R. (1994) 'The Milstar system'. doi: 10.2514/6.1994-1013.

[4] Curtis E. Lemay Center (2012) 'Offensive space control', p. 4.

[5] 'Anti-Satellite Weapons, Countermeasures, and Arms Control' (1985) Technology, (September).

[6] UK Ministry of Defence (2017) 'UK Air and Space Power', Joint Doctrine Publication 0.30, (Second Edition), p. 138.

[7] Lyon, H., Inalhan, G., Tsourdos, A. and Bourne, D. (2020) High-Altitude UAS Pseudo-Satellites: Architecture for End-to-End Military Communications. Cranfield University.

[8] BAE (2016) 'Linking the UK Armed Forces - Falcon Overview', BAE Systems.

[9] Tidswell, R. (2018) 'Airbus HAPS Zephyr Overview of Zephyr Short endurance - An Overview'.

[10] Valavanis, K. P. and Vachtsevanos, G. J. (2015) 'Handbook of unmanned aerial vehicles', Handbook of Unmanned Aerial Vehicles, pp. 1-3022. doi: 10.1007/978-90-481-9707-1.

[11] Ahmad, M. H. A. (2009) 'Minimizing Handoff Ping-Pong Effect in 802 . 11 Data Networks Using Differential RSS and Extrapolation', (1), pp. 28-38.

[12] Pahal, S. and Rathee, N. (2016) 'Analysis Of Hysteresis Margin For Effective Handover In 4g Wireless Networks Analysis Of Hysteresis Margin For Effective Handover In 4G Wireless Networks’, ICTACT Journal on Communication Technology, 7(4), pp. 1438-1442. doi: 10.21917/ijct.2016.0212.

[13] UK Ministry of Defence (2016) 'Joint Doctrine Publication 600 Communications and Information Systems Support To Joint Operations', Joint Doctrine Publication, (3).

[14] Danish Army Vehicles (2020) Mowag Duro III - Vehicle Summary, armyvehicles.dk. Available at: https://www.armyvehicles.dk/mowagduroIIIp.htm (Accessed: 10 April 2020).

[15] Lye, H. (2019) Second Airbus Zephyr UAS crashes during a test flight, www.airforce-technology.com. Available at: https://www.airforce-technology.com/news/second-airbus-zephyr-uas-crashes-during-a-test-flight/ (Accessed: 13 June 2020). 


\title{
Cranfield University
}

2021-01-04

\section{High-altitude UAS pseudo-satellites:} architecture for end-to-end military communication:

\author{
Lyon, Harry M.
}

AIAA

Lyon H, Inalhan G, Bourne D, Tsourdos A. (2021) High-altitude UAS pseudo-satellites:

architecture for end-to-end military communications. In: AIAA SciTech Forum 2021, 11-15 and 19-21 January 2021, Online

https://doi.org/10.2514/6.2021-0521

Downloaded from Cranfield Library Services E-Repository 